\title{
Mix it Up, Mix it Down Intriguing Implications of Ocean Layering
}

BY PETER J.S. FRANKS AND SHARON E.R. FRANKS

\section{PURPOSE OF ACTIVITY}

Using a physical simulation, we explore the vertical density structure within the ocean and how layering (density stratification) controls water motion, impedes nutrient transport, and regulates biological productivity. Our demonstration enables students to visualize the formation of horizontal layers in the ocean's interior and the slow undulation of large-amplitude internal waves. We show that stratification limits the vertical transport of energy and nutrients. The region of strong vertical density gradient-the pycnocline-is a barrier to the downward propagation of wind energy as well as the upward transport of nutrients into the euphotic zone.

\section{AUDIENCE}

Designed for graduate oceanography courses, this simulation is suitable for students as young as elementary school age, provided the level of discussion is appropriately scaled.

\section{BACKGROUND}

Density is measured as the mass per unit volume of a substance. The density of ocean water is controlled mainly by its salt content and temperature. The ocean's surface is warmed by the sun, and receives freshwater from precipitation and the land. The deep waters of the ocean, formed near the poles during winter, are cold and salty. Water at the surface of the Atlantic Ocean, for example, has a density about $1024 \mathrm{~kg} \mathrm{~m}^{-3}$. At the bottom of the ocean, 4000-m below, the density is about $1028 \mathrm{~kg} \mathrm{~m}^{-3}$-an increase of less than $0.5 \%$. Though the density difference seems small, it is fundamentally important to the circulation, mixing, and biological productivity of the ocean.
The density increase between the surface waters and the deep waters is often confined to a few relatively thin layers known as pycnoclines. Most ocean basins have a permanent pycnocline between about 500 and 1000-m depth, and a seasonal pycnocline that forms during the summer between about 20 and 100-m depth.

Small density differences between ocean layers reduce the gravitational force acting on a layer. The reduced gravity allows waves inside the ocean-internal waves - to achieve amplitudes of tens of meters. Internal waves move very slowly compared to the waves at the ocean's surface with which beachgoers are more familiar.

It takes a surprisingly large amount of energy to mix through even the small density differences across the pycnocline.

Thus, pycnoclines create effective barriers to vertical mixing, especially mixing forced by the wind blowing on the ocean surface. This barrier is particularly relevant to ocean plankton, as phytoplankton require light and nutrients to grow. Sunlight is most intense at the ocean's surface, but the nutrients are mostly below the pycnocline. The strength of the pycnocline thus determines the rate at which nutrients are supplied to the phytoplankton. Oceans with strong, permanent pycnoclines such as the North Pacific tend to have lower rates of productivity than oceans that mix strongly during the winter such as the North Atlantic (Parsons and Lalli, 1988).

Peter J.S. Franks (pfranks@ucsd.edu) is Professor of Biological Oceanography, Scripps Institution of Oceanography, La Jolla, CA, USA. Sharon E.R. Franks is an oceanographer, Scripps Institution of Oceanography, La Jolla, CA, USA. 


\section{DESCRIPTION OF THE ACTIVITY}

In a tabletop tank containing a layer of freshwater above a layer of salty water, students observe the formation of a third, intermediate-density layer. The third layer, colored with food dye, intrudes horizontally between the two original layers, generating an internal wave that moves more slowly and is of larger amplitude than waves at the air-water interface. Subsequent injection of food dye followed by a "wind event"-air blown across the water's surface-show that the pycnocline is a surprisingly effective barrier to mixing.

\section{Materials}

1. A rectangular, transparent tank with no lip around the inside upper edge. We use a 30 -in $\mathrm{L} x$ 12-in $\mathrm{H}$ x 8-in W acrylic tank, but the dimensions are not too important.

2. A barrier that can be inserted vertically to seal off one part of the tank from another. The barrier must be easy to remove without disturbing the water too much.

3. Freshwater to fill almost half the tank.

4. Salty water to fill almost half the tank, preferably in a container with a spigot near the bottom. We add about 1/4 cup of salt to $\sim 3$ gallons of freshwater, but the exact proportions are not important.

5. Plastic tubing long enough to extend from the elevated container of saltwater to the bottom of the tank, approximately $3 \mathrm{ft}$. The diameter is not too important, but the internal diameter should be less than about .25 in.

6. A clamp or valve for the tubing.

7. Duct tape.

8. Two or three colors of food coloring.

9. A long pipette or eyedropper long enough to reach the bottom of the tank.

10. Something to stir with-for example, a spoon, fork, ruler, spatula.

11. Some cups or tubs to catch drips and splashes.

12. Paper towels.

Tank setup will take approximately 45 minutes. The demonstrations can take from 10 minutes to half an hour, depending on the amount of discussion and questioning.

\section{Setting Up the Tank}

Place the empty tank where you will conduct the demonstration. Fill the tank half full of freshwater, and let the water settle for a few moments. Place the saltwater container beside the tank on a support (e.g., a chair or some books) to raise it above the tank. With one end of the tubing connected to the saltwater container as either a siphon or attached to the spigot, and the other end of the tubing in a sink or waste container, allow the tube to fill completely with saltwater, expelling any air bubbles in the tubing, as they will cause undesirable mixing during the filling of the tank. The saltwater should trickle out of the hose at about 2-5 $\mathrm{ml}$ per second. At this rate, it will take about 45 seconds to fill an $8-\mathrm{oz}$ cup. If the water comes out too fast, the stratification will be weak, and the demonstration will not be very dramatic.

With your finger momentarily blocking the flow of saltwater, and taking care to disturb the water in the tank as little as possible, position the blocked end of the saltwater tubing in the bottom of the freshwater-filled tank, near one end of the tank, with the trickle pointing toward the other end. Carefully remove your hand, allowing the flow of saltwater to resume. To ensure that the tube stays in position during filling, tape the dry, upper part of the tube to the side of the tank.

As the tank is filling, you will be able to see the freshwater/ saltwater interface. It is most visible from the side of the tank, with eye level near the fresh-salt interface. The interface will slowly wave up and down, showing the billows of turbulence. Once the fresh-salt interface is above the level of the hose outlet, you can increase the flow rate a little. The goal is to mix as little as possible during the filling of the tank, which will take 15-30 minutes, depending on the size of the tank.

Once you have about equal volumes of fresh and saltwater in the tank, carefully remove the hose.

\section{PART 1: INTERNAL WAVE AND DENSITY STRATIFICATION}

Place the barrier vertically in the tank, dividing it into a small volume at one end that is about one-fifth (or less) the volume of the tank. Add a few drops of food coloring to the water in the small-volume end, and mix it thoroughly with a spoon, spatula, or other implement.

Ask the students: What do you think will happen when I remove the barrier? Typical answers include: The colored water will flow to the top. The colored water will flow to the bottom. 
The colored water will mix with the clear water.

In one smooth motion, remove the barrier. The colored water will bulge toward the middle depths of the tank, then neck into a layer that gradually propagates to the far end of the tank, sloshes up the wall seemingly in slow motion, and then washes back (Figure 1). Point out how slowly the wave is moving, and its large amplitude relative to the amplitude of waves one sees at the air-water interface. Make some faster-moving, loweramplitude surface waves by wiggling your finger at the water's surface. Invite the students to compare the surface and internal waves. Point out that slow speed and large amplitude are characteristic of motions in the ocean.

Explain that vertical displacements of water in the ocean are returned to their original position by gravity. However, the effect of the gravity is reduced by the small density differences between the adjacent layers of water. In the ocean's interior, the density gradient is very small, so the restoring force is very weak. At the surface, the density gradient from the water to the air is very large. The strong restoring force creates waves of relatively small amplitude that move very quickly. Inside the ocean, the waves tend to be about ten times the amplitude of surface waves, and move at only one-tenth the speed.

Now ask the class: Why did the colored water move through the tank the way it did? The answers, often creative, have included a suggestion that the food coloring somehow stuck the water molecules together. In our graduate courses, someone will usually comment that the density of the colored water is different than the water above and below it.
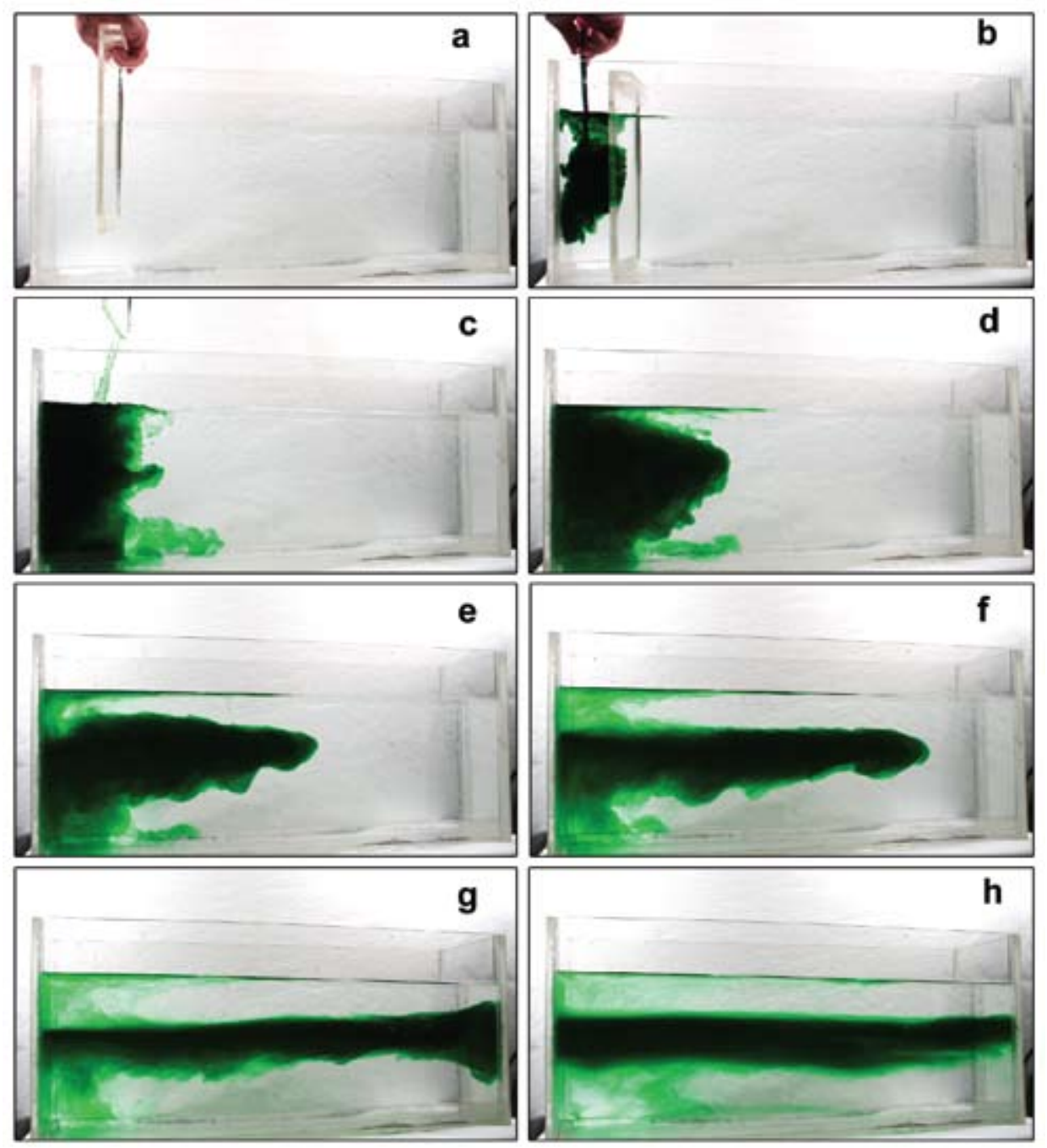

Figure 1. (a) Placing the barrier in the tank. (b) Stirring the food coloring. (c) Removing the barrier. $(d-g)$ The colored intermediate-density water forming a new layer below the upper freshwater layer, and above the lower saltwater layer. This intrusion took about 20 seconds. (h) The tank several minutes after removal of the barrier and all the internal waves have died down.
To motivate the correct interpretation, it can be helpful to review the basics of density. Ask the students: Which would weigh more: freshwater, or an equal volume of salty water? Imagine a cup of freshwater on a scale and adding salt to it. Intuition tells us that the resultant saltwater will weigh more than the freshwater. Point out that a liter of ocean water contains about $25 \mathrm{~g}$ of salt.

Then, ask: What happens if we mix some freshwater and some salty water? Students usually correctly surmise that the mixed water will be of salinity-and density-between the two waters being mixed.

To put these ideas in an oceanographic context, ask: Based 
on what we've just discussed, where would you expect to find cold, salty water in the ocean? How about warm freshwater? Answers to these questions usually lead to the idea of a stratified ocean, with warmer, fresher water near the surface, and colder, saltier water near the bottom.

Returning to the tank, tell the students that you had set up the tank with a layer of freshwater at the surface and a layer of salty water below. Ask if someone would come up to the board and draw the vertical profile of density in the tank before the barrier was inserted. With luck, you'll get something that looks like Figure 2a. Ask a student to draw a line showing the density of the water in the well-mixed region that was barricaded from the rest of the tank (Figure 2a, green line). Finally, ask a student to draw the profile of the density in the tank as it looks now. It should look something like Figure $2 b$, with a third layer of intermediate-density sandwiched between the upper and lower layers.

Now explain that when you barricaded the small volume of water at the end of the tank and mixed it, you created a water mass that had a density that was greater than the freshwater, but less than the salty water. When the barrier was removed, this water had to sink below the freshwater layer at the surface, but float on top of the salty layer at the bottom. The only place it could go was into a new layer formed between the fresh and salty layers.

\section{PART 2:}

\section{BARRIER TO MIXING}

The layer of colored water and the clear layers immediately above and below it are a region in which the density changes vertically. The region of strong density change is called the "pycnocline," from the Greek words "pycnos" meaning "density" and "cline" meaning "slope." A pycnocline formed by temperature changes is a "thermocline," while a pycnocline formed by salinity changes is a "halocline." Pycnoclines in the ocean are usually both thermoclines and haloclines, while those in lakes are thermoclines.

Tell the class that you are now going to demonstrate how a pycnocline can be a barrier to vertical mixing. Motivate interest in vertical mixing by introducing the needs of ocean primary producers-the phytoplankton.

Explain that phytoplankton, like plants on land, need light and nutrients to grow. In the ocean, phytoplankton live near the ocean surface where sunlight penetrates the water. But, because the phytoplankton continuously use up the nutrients in the surface waters, the upper ocean is relatively depleted in nutrients compared with the deeper waters, below the pycnocline. Ask the students: How do nutrients get from the deep waters up to the surface where there's enough light for the phytoplankton to grow?

Among the ideas students usually propose is vertical mixing - that tides, other ocean currents, or wind mix the water, driving nutrient-rich deep water upwards. Point out that if there is a pycnocline, vertical mixing requires denser water to be brought upward, and less-dense water to be pushed downward. This mixing requires energy. The energy for mixing often comes from the wind blowing on the surface of the ocean.

Take a cup and put a little water in it, and a few drops of food coloring. (Use a new color.) Using the pipette or eyedropper, make a vertical streak of this colored water near the 

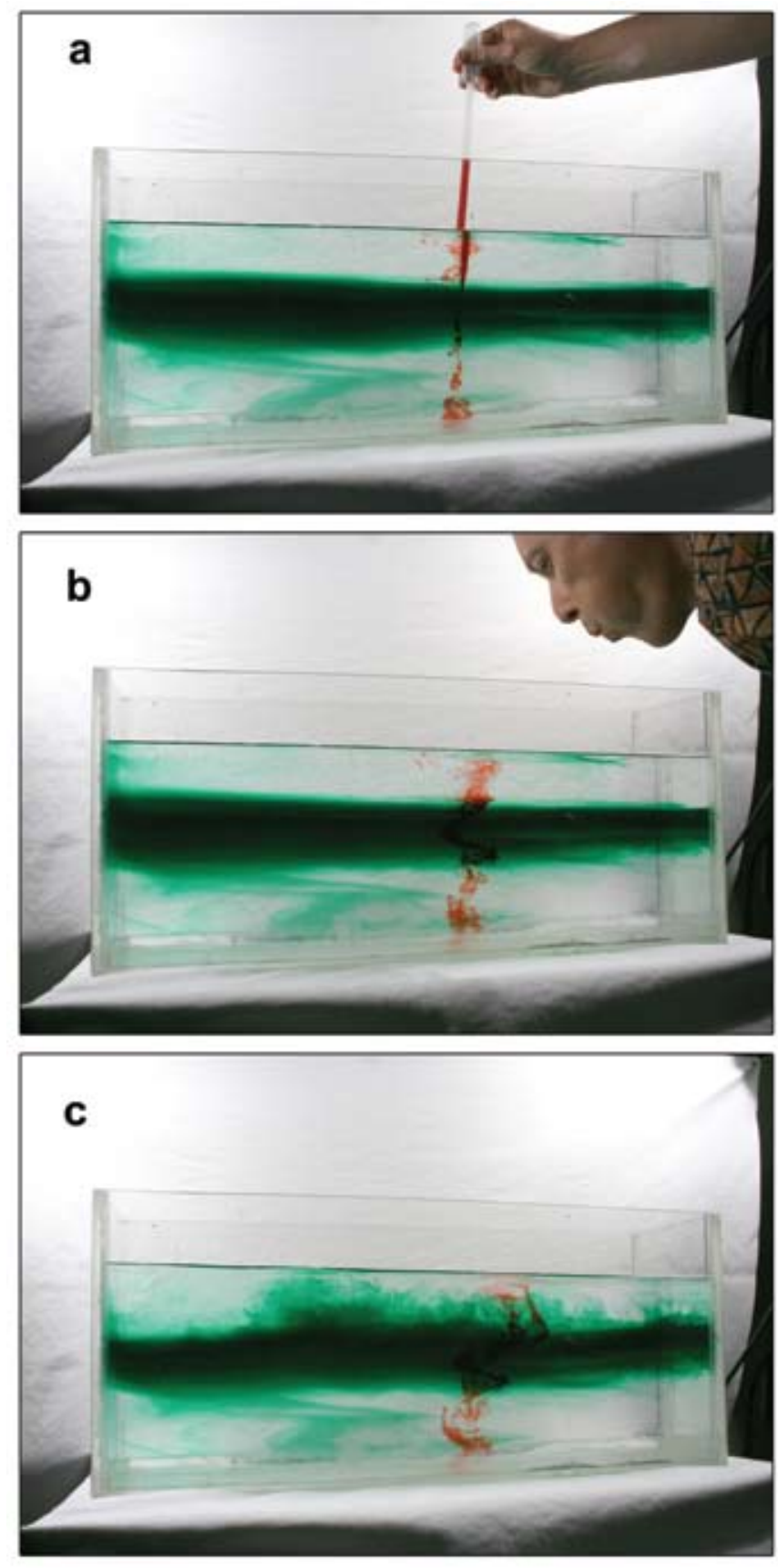

Figure 3. Effects of wind mixing. (a) Forming a vertical streak of red dye in the tank. (b) Blowing on the surface of the water. (c) The dye at the top of the pycnocline is mixed upward by turbulence caused by the wind. The dye below the pycnocline, however, remains unmixed. The blowing will also create internal waves at the pycnocline.

middle of the tank, beginning near the bottom and drawing the pipette upward. As you draw the streak, it is helpful to suck a little water from the tank into the pipette and make a column of little colored puffs. The mixing in the pipette will allow the colored water to be closer to the density of the fluid around it, so it won't float up to the surface.
Invite a student who guessed wind mixing to come to the tank. Tell the class that this person is a hurricane, who is going to try to mix some nutrients up to the phytoplankton.

Ask the volunteer to blow as hard as possible on the surface of the tank. The dye streak will mix with the surrounding water in the surface layer, but remain unmixed below the pycnocline (Figure 3). The tank we use allows a surface layer about 3-4 in thick, and it is almost impossible to blow hard enough to mix the dye streak below the pycnocline. Explain that there is not enough energy in the wind to lift the dense water upward. This is a good example of what happens in the ocean: it takes a very strong storm to actually mix through the pycnocline. The density gradient is just too strong for the amount of energy available from the wind. The persistent pycnocline causes the phytoplankton to be nutrient-limited for much of the year.

\section{RELEVANCE TO OCEANOGRAPHY}

Ocean stratification exerts a fundamental control over the ocean's physical motions, as well as its chemical and biological dynamics. One of the main differences between the North Atlantic and the North Pacific, for example, is that the North Atlantic mixes deeply (to $400 \mathrm{~m}$ or more) in the wintertime, whereas the North Pacific remains stratified and mixes only to about $200 \mathrm{~m}$ (e.g., Parsons and Lalli, 1988). The permanent stratification in the North Pacific reduces the upward mixing of nutrients, creating a situation in which the phytoplankton are nutrient-limited all year round. In the North Atlantic, restratification of the waters in the springtime (due to solar heating) creates a shallow pycnocline and a surface layer that is rich in dissolved nutrients. This shallow pycnocline allows the phytoplankton to obtain enough light for growth; with the abundant nutrients, the phytoplankton form a dense bloom that can last a month or more. This "spring bloom" is not seen in the North Pacific, as the pycnocline there forms a permanent barrier to the upward mixing of nutrients.

These different stratifications in the North Atlantic and North Pacific also drive differences in the organisms that eat the phytoplankton. The dominant crustacean zooplankton of the North Atlantic (the copepod Calanus finmarchicus) have quite different life cycles than the dominant crustacean zooplankton of the North Pacific (the copepod Neocalanus plumchrus). The phytoplankton and zooplankton of the North Pacific tend to be dominated by smaller organisms than in the North Atlantic. This size difference between the two oceans is 
also a consequence of the reduced nutrient flux in the North Pacific, caused by the permanent pycnocline.

The activities described here should serve as memorable examples of the importance of subtle density gradients on the physical and biological dynamics of the world's ocean. This introduction to the sometimes-surprising effects of density changes can be expanded with further activities exploring diffusion and mixing (e.g., Karp-Boss et al., 2007), fronts (e.g., Nadiga and Aurnou, 2008), and the global thermohaline circulation.

\section{ACKNOWLEDGEMENTS}

Thanks are due to Matt Soave and Corey Sheredy who took the photos, and to Phil Hastings and H.J. Walker who allowed us to use their lab and lights during the photography. Particular thanks are due to Ryan Willis who filmed and produced the video made to accompany this lesson. We also thank Lee Karp-Boss and Ellen Kappel for their reviews of the manuscript. 还

\section{ADDITIONAL ONLINE RESOURCES}

Videos of these demonstrations suitable for classroom use can be found at the following URLs (video is 11 minutes and 20 seconds long):

- Mac low-resolution version (80 Mb): http://spiff.ucsd.edu/mixitup_web_mac.mov

- Mac high-resolution version (222 Mb): http://spiff.ucsd.edu/mixitup_big.mov
- Windows Media Viewer version (122 Mb): http://spiff.ucsd.edu/mixitup_windows.wmv

\section{Density}

- http://www.visionlearning.com/library/ module_viewer.php?mid=37

- http://www.onr.navy.mil/focus/ocean/water/default.htm

- http://topex-www.jpl.nasa.gov/education/activities/ ts1 siac2.pdf

- http://www.windows.ucar.edu/tour/link=/earth/ Water/density.html

- http://www.teachertube.com/ view_video.php?viewkey=8cfa3ed9b1d0a4a8938e

\section{Thermohaline Circulation}

- http://www.youtube.com/watch?v=FuOX23yXhZ8

\section{REFERENCES}

Karp-Boss, L., E. Boss, and J. Loftin. 2007. Diffusion at work: An interactive simulation. Oceanography 20(3):127-131. Available online at: http://tos.org/hands-on/ activities/20.3_karp-boss_et_al.pdf (accessed January 21, 2009).

Nadiga, B.T., and J.M. Aurnou. 2008. A tabletop demonstration of atmospheric dynamics: Baroclinic instability. Oceanography 21(4):196-201. Available online at: http://tos.org/oceanography/issues/issue_archive/issue_pdfs/21_4/21.4_ nadiga.pdf (accessed January 21, 2009).

Parsons, T.R., and C.M. Lalli. 1988. Comparative oceanic ecology of the plankton communities of the subarctic Atlantic and Pacific oceans. Pp. 317-359 in Oceanography and Marine Biology: An Annual Review, Volume 26. H. Barnes and M. Barnes, eds, Aberdeen University Press.

\section{HANDS-ON OCEANOGRAPHY}

Hands-On Oceanography provides an opportunity for you to publish teaching materials developed for undergraduate and/or graduate classes in oceanography. Activities include, but are not limited to, computer-based models and laboratory demonstrations that actively engage students (i.e., activities where students have to make decisions, record results, and interpret results). All submissions are peer-reviewed. Publication of teaching materials may contribute to the broader impact of NSF-funded research.

Visit www.tos.org/hands-on to download

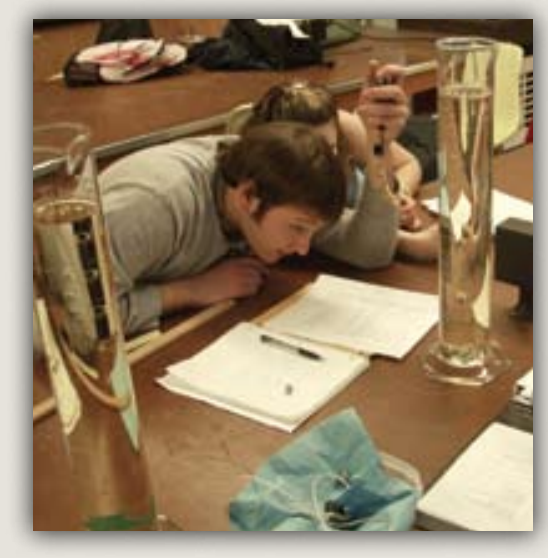
activities or for more information on submitting an activity of your own for consideration. 